\title{
Necessity and Insufficiency of Scale Invariance for solving Cosmological Constant Problem
}

\author{
Taichiro Kugo* \\ Yukawa Institute for Theoretical Physics, Kyoto University, Kyoto 606-8502, Japan \\ E-mail: kugo@yukawa.kyoto-u.ac.jp
}

\begin{abstract}
A scenario based on the scale invariance for explaining the vanishing cosmological constant (CC) is discussed. I begin with a notice on the miraculous fact of the $\mathrm{CC}$ problem that the vacuum energies totally vanish at each step of hierarchical and successive spontaneous symmetry breakings. I then argue that the classical scale invariance is a necessary condition for the calculability of the vacuum energy.

Next, I discuss how sufficient the scale invariance is for solving the CC problem. First in the framework of classical field theory, the scale invariance is shown to give a natural mechanism for realizing the miracle of vanishing vacuum energies at every step of spontaneous symmetry breakings. Then adopting Englert-Truffin-Gastmans' prescription to maintain the scale invariance in quantum field theory, I point out that the quantum scale invariance alone is not yet sufficient to avoid the superfine tuning of coupling constants for realizing vanishingly small cosmological constant, whereas the hierarchy problem may be solved. Another symmetry or a mechanism is still necessary which protects the flat direction of the potential against the radiative corrections.
\end{abstract}

Corfu Summer Institute 2019 "School and Workshops on Elementary Particle Physics and Gravity" (CORFU2019)

31 August - 25 September 2019

Corfù, Greece

\footnotetext{
* Speaker.
} 


\section{Introduction}

Cosmological constant problem is a dark cloud hanging over the two well-established theories Quantum Field Theory $\Longleftrightarrow$ Einstein Gravity Theory.

I first explain my viewpoint on what is actually the problem.

Presently observed Dark Energy $\Lambda_{0}$ looks like a small Cosmological Constant (CC):

$$
\text { Present observed CC: } \quad 10^{-29} \mathrm{gr} / \mathrm{cm}^{3} \sim 10^{-47} \mathrm{GeV}^{4} \sim(1 \mathrm{meV})^{4} \equiv \Lambda_{0} .
$$

I do not try to explain this tiny CC now, since it will eventually be explained after our CC problem is solved. However, we use it as the scale unit $\Lambda_{0}$ of our discussion in this Introduction.

Now, from my viewpoint, the essential point of the CC problem is the following miraculous fact; that is, there are several dynamical symmetry breakings in this world and they are all accompanied by vacuum condensation energies, ranging over wide and hierarchical scales. Nevertheless, those vacuum condensation energies are almost completely canceled at each stage of those spontaneous symmetry breakings.

From the success of the Standard Model, in particular, we are confident of the existence of at least two symmetry breakings:

$$
\begin{array}{r}
\text { Higgs Condensation : }-V_{\mathrm{Higgs}} \sim(200 \mathrm{GeV})^{4} \sim 10^{9} \mathrm{GeV}^{4} \sim 10^{56} \Lambda_{0}, \\
\text { QCD Chiral Condensation }\langle\bar{q} q\rangle^{4 / 3}:-V_{\mathrm{QCD}} \sim(200 \mathrm{MeV})^{4} \sim 10^{-3} \mathrm{GeV}^{4} \sim 10^{44} \Lambda_{0} .
\end{array}
$$

These are $10^{56}$ and $10^{44}$ times larger, respectively, than the present CC value $\Lambda_{0}$. Nevertheless, the fact that our calm universe exists means that these surely existing vacuum energies are not contributing to the $\mathrm{CC}$ at all! That is, Einstein gravity does not feel these condensation energies at all. If these condensation energies are canceled by an initially prepared "bare cosmological constant" $c$, then, even these two spontaneous breakings alone imply that the cancellation must occur exactly over more than 56 digits. If we rephrase this fact more vividly, then, the Higgs condensation energy $V_{\mathrm{Higgs}}$ and chiral condensation energy $V_{\mathrm{QCD}}$ are, respectively, canceled by the bare $\mathrm{CC}$ value $c$ exactly by 12 digit and 44 digit of concrete numbers, respectively, as shown as follows:

$$
\begin{aligned}
& c \text { (initially prepared CC) } \\
& =\underbrace{654321,098765}_{12 \text { digits }} 4321,0987654321,0987654321,0987654321,0987654321 \times \Lambda_{0} \sim 10^{56} \Lambda_{0} \\
& c+V_{\mathrm{Higgs}}=\underbrace{4321,0987654321,0987654321,0987654321,0987654321}_{44 \text { digits }} \times \Lambda_{0} \sim 10^{44} \Lambda_{0} \\
& c+V_{\mathrm{Higgs}}+V_{\mathrm{QCD}}=\quad \text { present Dark Energy: } 1 \times \Lambda_{0} \sim \Lambda_{0}
\end{aligned}
$$

Note that the vacuum energy is almost totally canceled at each stage of spontaneous symmetry breaking as far as in the order of the relevant energy scale.

In this talk, I would like to propose the classical scale invariance as an essential ingredient for solving the $\mathrm{CC}$ problem. Here the classical scale invariance means that the theory has no dimensionful parameters at all. Indeed in Section 2, I give an argument that the classical scale 
invariance is a necessary condition for the calculability of the vacuum energy. Otherwise the theory must have a bare cosmological constant term as a free parameter UV counterterm, implying that there is no hope to determine its renormalized value by calculation in the theory.

In later Sections 3 to 5, we will discuss how the scale invariance is sufficient to solve the CC problem. In subsection 3.1, we discuss the problem in a classical field theory framework, namely at tree level in quantum field theory. There I present a scale invariant model possessing a suitable potentials, and show that the scale invariance gives a natural mechanism for realizing the miracle of vanishing vacuum energies at every step of the successive spontaneous symmetry breakings. Then, moving to quantum theory in subsections 3.2 and 3.3, I explain the Englert-Truffin-Gastmans' [1] prescription to maintain the scale invariance in quantum field theory. In Section 4, we discuss the quantum scale invariant renormalization explicitly for Schaposhnikov-Zenhausern's model[2] of two scalar fields, Higgs and dilaton fields, whose characteristic energy scales are $10^{2} \mathrm{GeVand}$ $10^{18} \mathrm{GeV}$, respectively. Based on the explicit computations by Ghilencea[15], we will see that the hierarchy is maintained stable against the radiative corrections. However, I will point out that we actually need superfine tuning of coupling constants to realize the vanishingly small vacuum energy, implying reappearance of CC problem. The puzzle why the quantum scale invariance does not automatically guarantees the vanishing vacuum energy is resolved in Section 5. In Section 6 , we discuss in more detail and generally how the hierarchy problem is solved in quantum scale invariant theory even including gravity loop corrections. Section 7 is devoted to conclusion.

\section{Scale Invariance is a Necessary Condition}

We show in this section that the classical scale invariance is a necessary condition for the CC problem to be solvable. For preparation for it, we first have to clear up a possible confusion about the vacuum energy.

\section{1 quantum vacuum energy $\simeq$ potential energy}

People may suspect that there are two distinct sources for the cosmological constant. One is the vacuum energy in quantum field theory, zero-point oscillation energy for boson fields and negative energy in the Dirac sea for fermions,

$$
\text { (Quantum) Vacuum Energy } \quad \sum_{k, s} \frac{1}{2} \hbar \omega_{k}-\sum_{k, s} \hbar E_{k}
$$

which is divergent in nature and usually simply discarded. Another is the potential in classical field theory:

$$
\text { (Classical) Potential Energy } \quad V\left(\phi_{c}\right) \text { : potential }
$$

which is finite in nature and gives the vacuum condensation energy in the case of spontaneous symmetry breaking. These two are separately stored in our (or my, at least) memory, but actually, almost the same object, as we now see.

We now show for the vacuum energies in the Standard Model (SM) that

$$
\text { quantum Vacuum Energy } \simeq \text { Higgs Potential Energy }
$$


To see this more explicitly, let us consider a simplified (analogue of) SM:

$$
\begin{aligned}
\mathscr{L}_{\mathrm{r}}= & \bar{\psi}\left(i \gamma^{\mu} \partial_{\mu}-y \phi(x)\right) \psi(x) \\
& +\frac{1}{2}\left(\partial^{\mu} \phi(x) \partial_{\mu} \phi(x)-m^{2} \phi^{2}(x)\right)-\frac{\lambda}{4 !} \phi^{4}(x)-h m^{4} .
\end{aligned}
$$

Here, $\phi$ is a single component scalar field as an analogue of Higgs field, and $\psi$ is a Dirac fermion as an analogue of quark/lepton fields whose mass comes solely from the non-vanishing vacuum expectation value (VEV) of Higgs, $\langle\phi\rangle \neq 0$. The last term $-h m^{4}$ is the vacuum energy (CC) term.

Effective action and effective potential are calculated prior to the vacuum choice (i.e., calculable independently of the choice of the vacuum). The effective potential $V(\phi)$ at 1-loop level in this simplified SM is given in the following well-known form :

$$
\begin{aligned}
V\left(\phi, m^{2}\right) & =V_{\text {tree }}+V_{1-\text { loop }}+\delta V_{\text {counterterms }}^{(1)} \\
V_{\text {tree }} & =\frac{1}{2} m^{2} \phi^{2}+\frac{\lambda}{4 !} \phi^{4}+h m^{4} \\
V_{1-\text { loop }} & =\frac{1}{2} \int \frac{d^{4} k}{i(2 \pi)^{4}} \ln (-k^{2}+\underbrace{m^{2}+\frac{1}{2} \lambda \phi^{2}}_{=M_{\phi}^{2}(\phi)})-2 \int \frac{d^{4} p}{i(2 \pi)^{4}} \ln (-p^{2}+\underbrace{y^{2} \phi^{2}}_{=M_{\psi}^{2}(\phi)}) .
\end{aligned}
$$

The 1-loop integral is evaluated in the dimensional regularization. Using dimensional formula

$$
\frac{1}{2} \mu^{4-n} \int \frac{d^{n} k}{i(2 \pi)^{n}} \ln \left(-k^{2}+M^{2}\right)=\frac{M^{4}}{64 \pi^{2}}(-\frac{1}{\bar{\varepsilon}} \underbrace{+\ln \frac{M^{2}}{\mu^{2}}-\frac{3}{2}}),
$$

Coleman-Weinberg potential

and dropping the $1 / \bar{\varepsilon}$ parts in $\overline{\mathrm{MS}}$ renormalization scheme $\left(\frac{1}{\bar{\varepsilon}}=\frac{1}{\varepsilon}-\gamma+\ln 4 \pi, \varepsilon=2-\frac{n}{2}\right)$, we obtain finite well-known renormalized 1-loop effective potential:

$$
\begin{aligned}
V\left(\phi, m^{2}\right)= & \frac{1}{2} m^{2} \phi^{2}+\frac{\lambda}{4 !} \phi^{4}+h m^{4} \\
& +\frac{\left(m^{2}+\frac{1}{2} \lambda \phi^{2}\right)^{2}}{64 \pi^{2}}\left(\ln \frac{m^{2}+\frac{1}{2} \lambda \phi^{2}}{\mu^{2}}-\frac{3}{2}\right)-4 \frac{(y \phi)^{4}}{64 \pi^{2}}\left(\ln \frac{y^{2} \phi^{2}}{\mu^{2}}-\frac{3}{2}\right)
\end{aligned}
$$

Note that the divergences $\propto 1 / \bar{\varepsilon}$ appear in the terms proportional to

$$
\begin{aligned}
& M_{\phi}^{4}(\phi)=\left(m^{2}+\frac{\lambda}{2} \phi^{2}\right)^{2}=m^{4}+\lambda m^{2} \phi^{2}+\frac{\lambda^{2}}{4} \phi^{4} \text { and to } \\
& M_{\psi}^{4}(\phi)=(y \phi)^{4}=y^{4} \phi^{4} .
\end{aligned}
$$

These divergences proportional to $\phi^{4}, m^{2} \phi^{2}$ and $m^{4}$ are renormalized into $\lambda, m^{2}$ and $h$, respectively. Here we should recall the fact that these 1-loop contributions of the boson and fermion loops are just the same object as the quantum vacuum energies mentioned above in Eq. 2.1], namely, zeropoint oscillation energy for boson fields and negative energy in the Dirac sea for fermions. They are divergent but are renormalized into the parameters $\lambda, m^{2}$ and $h$. The main part of quantum vacuum energies are already included in the classical potential $V_{\text {tree }}(\phi)$ with renormalized parameters $\lambda, m^{2}$ and $h$, since the 1-loop parts (i.e., Coleman-Weinberg potential parts) are small corrections to the renormalized tree level potential at energy scale around the renormalization point $\phi \sim \mu$. 


\section{2 conclusions from these simple observations}

From this simple observation, we can draw very interesting and important conclusions. As far as the matter fields and gauge fields are concerned in the SM, we note that their masses solely come from the Higgs condensation $\langle\phi\rangle$, so

the quantum vacuum energies coming from the matter and gauge fields are calculable and finite quantities in terms of the renormalized $\lambda$ parameters.

This is because their masses $M$ are proportional to Higgs VEV $\phi$, and the divergences of their vacuum energies are proportional to $\phi^{4}$ (at 1-loop, at least.)

However, the Higgs field itself is an exception! The divergences of the Higgs vacuum energy are not only $m^{2} \phi^{2}$ and $\phi^{4}$ but also the zero-point function proportional to $m^{4}$. This comes from the right diagram in Figure 1. The left diagram proportional to $m^{2}$ vanishes as far as we use dimensional regularization. In order to cancel that part, we have to prepare the bare vacuum energy $(\mathrm{CC})$ term
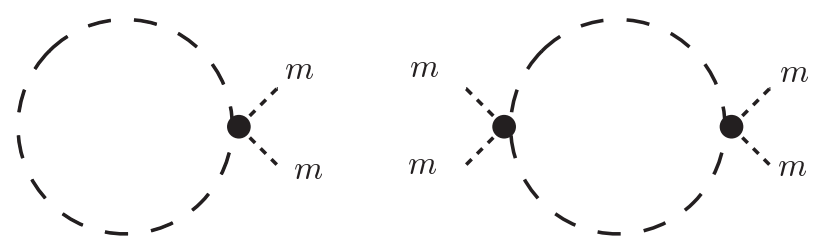

Figure 1: Divergent vacuum energy diagrams coming from the Higgs loop, where the dotted line represents the massless Higgs propagators.

$h_{0} m_{0}^{4}$ from the beginning to yield the counterterm:

$$
h_{0} m_{0}^{4}=Z_{h} Z_{m}^{2} h m^{4}=(1+F) h m^{4} \quad \rightarrow \quad F^{(1)} h=\frac{1}{64 \pi^{2}} \frac{1}{\bar{\varepsilon}} .
$$

Then, the renormalized CC term $\mathrm{hm}^{4}$ becomes a free parameter. This implies that there is no chance to explain the value of $C C$.

We thus reach an important conclusion:

For the calculability of CC, we should have $m^{2}=0$, or equivalently no dimensionful parameters in the theory $\Rightarrow$ (Classical) Scale-Invariance.

\section{Scale Invariance may solve the CC Problem}

Our world is almost scale invariant: that is, the SM Lagrangian is scale invariant except for the Higgs mass term. So if the Higgs mass term comes from the spontaneous breaking of scale invariance at higher energy scale physics, the total system can really be scale invariant (classically, at least):

$$
\lambda\left(h^{\dagger} h-m^{2}\right)^{2} \rightarrow \lambda\left(h^{\dagger} h-\varepsilon \Phi^{2}\right)^{2} .
$$

where $h$ is Higgs field and $\Phi$ is a certain scalar field relevant to the higher energy physics; for instance, $\Phi$ may be a field appearing in front of the Einstein-Hilbert term as

$$
\int d^{4} x \sqrt{-g} \Phi^{2} R
$$


We call this field $\Phi$ dilaton henceforth since it becomes the Nambu-Goldstone boson for spontaneous breaking of scale (= dilatation) invariance by its non-vanishing VEV.

I will explain in this section how the classical scale invariance (SI) may solve the CC problem; in particular, it would give a natural mechanism why the vacuum energy remains vanishing at every stage of hierarchical successive spontaneous symmetry breakings. Similar ideas have been proposed so far by many authors including Shaposhnikov and Zenhausern[2], Antoniadis and Tsamis[3], Tomboulis[4], Wetterich[5], and others [6, 7, 8, 9, 10]. My scenario is most similar to Shaposhnikov and Zenhausern[2], but no one has ever pointed out that it gives a natural mechanism for realizing vanishing vacuum energy at every stage of successive spontaneous symmetry breakings.

Before explaining my scenario using global SI, let me mention to the work by Antoniadis and Tsamis [3] and Tomboulis [4], whose papers appear very early and actually contain almost all basic ideas in this direction for solving the CC problem. Nevertheless those work use local SI which I think has to be useless:

\section{Local SI theory with dilaton (without Weyl gauge field) is meaningless.}

The reason is the following. If the dilaton field $\Phi_{0}(x)$ with dimension one is present, any action can be cast into local SI form so that the local SI itself means nothing: Indeed, for any given action $S[\phi]$ which may contain any mass terms, we can replace any fields $\phi_{i}$ with dimension $d_{i}$ by the scale invariant fields

$$
\phi_{i} \rightarrow \Phi_{0}^{-d_{i}} \phi_{i}=: \phi_{i}^{\prime}
$$

Then the action becomes local scale invariant under $\phi_{i}(x) \rightarrow \lambda(x)^{d_{i}} \phi_{i}(x)$ and $\Phi_{0}(x) \rightarrow \lambda(x) \Phi_{0}(x)$. But this local symmetry is fake since the system reduces to the original action $S[\phi]$ in the unitary gauge $\Phi_{0}(x)=1$. q.e.d.

Essentially the same but more detailed discussion was given by Tsamis and Woodard[11] for the conformal scalar-metric gravity theory. Note, however, that this argument applies only to the local SI system in which Weyl gauge field is absent. If the Weyl gauge field exists in the system, the gauge fixing cannot eliminate all four components of the Weyl gauge field [12, 13].

So, by SI henceforth, we always mean global scale invariance, or equivalently, the absence of dimensionful parameters in this paper.

\section{1 classical scale invariance: a possible scenario}

Suppose that our world has no dimensionful parameters. Let the effective potential $V$ of the total system look like

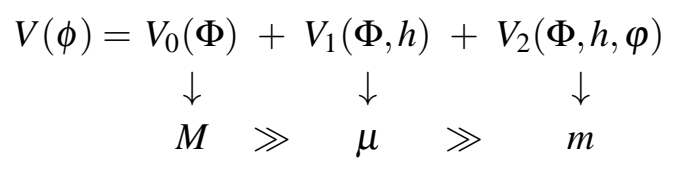

We suppose that $V_{0}(\Phi), V_{1}(\Phi, h)$ and $V_{2}(\Phi, h, \varphi)$ are relevant to the physics at three energy scales, Planck scale $M$, electroweak scale $\mu$ and QCD scale $m$, respectively, although they contain no 
dimensionful parameters. We also suppose that $h$ and $\varphi$ are Higgs field and chiral $S U(2)$ sigmamodel field, respectively. Then, classically, it satisfies the scale invariance relation :

$$
\sum_{i} \phi^{i} \frac{\partial}{\partial \phi^{i}} V(\phi)=4 V(\phi)
$$

with $\phi_{i}$ representing all the relevant scalar fields collectively. This implies that the vacuum energy vanishes at any stationary point $\left\langle\phi_{i}\right\rangle=\phi_{i}^{0}$ :

$$
V\left(\phi^{0}\right)=0
$$

Important point is that this holds at every stage of spontaneous symmetry breakings as far as the potential $V_{0}(\Phi), V_{0}(\Phi)+V_{1}(\Phi, h)$ and $V_{0}(\Phi)+V_{1}(\Phi, h)+V_{2}(\Phi, h, \varphi)$ are separately scale invariant (i.e., of dimension 4). This should be so because, for instance, we can retain only $V_{0}(\Phi)$ part when discussing the physics at scale $M$ since $h$ and $\varphi$ are expected to get much smaller VEVs of order $\mu$ or lower. Then the scale invariance guarantees $V_{0}\left(\Phi_{0}\right)=0$. This thus gives a very natural mechanism for realizing the miracle that the vacuum energy remains vanishing at every step of spontaneous symmetry breakings.

We can now write a toy model of potentials. First part is

$$
V_{0}(\Phi)=\frac{1}{2} \lambda_{0}\left(\Phi_{1}^{2}-\varepsilon_{0} \Phi_{0}^{2}\right)^{2}
$$

in terms of two real scalar fields $\Phi_{0}$ and $\Phi_{1}$, to realize VEVs

$$
\left\langle\Phi_{0}\right\rangle=M \quad \text { and } \quad\left\langle\Phi_{1}\right\rangle=\sqrt{\varepsilon_{0}} M \equiv M_{1} .
$$

This $M$ is totally spontaneous and there is no meaning in its magnitude at this stage. Only meaningful is whether it vanishes or not. We suppose $M$ be Planck mass giving the Newton coupling constant via the scale invariant Einstein-Hilbert term

$$
S_{\mathrm{eff}}=\int d^{4} x \sqrt{-g}\left\{c_{1} \Phi_{0}^{2} R+c_{2} R^{2}+c_{3} R_{\mu v} R^{\mu v}+\cdots\right\} .
$$

If the grand unified theory (GUT) stage exists, $\varepsilon_{0}$ may be a constant as small as $10^{-4}$ and then $\Phi_{1}$ gives the scalar field which breaks GUT symmetry; e.g., $\Phi_{1}: 24$ causing $S U(5) \rightarrow S U(3) \times$ $S U(2) \times U(1)$.

$V_{1}(\Phi, h)$ part causes the electroweak symmetry breaking:

$$
V_{1}(\Phi, h)=\frac{1}{2} \lambda_{1}\left(h^{\dagger} h-\varepsilon_{1} \Phi_{1}^{2}\right)^{2}
$$

with very small parameter $\varepsilon_{1} \simeq\left(10^{2} \mathrm{GeV} / 10^{16} \mathrm{GeV}\right)^{2} \simeq 10^{-28}$. This reproduces the Higgs potential when $h$ is the Higgs doublet field and $\varepsilon_{1} \Phi_{1}^{2}$ term is replaced by the VEV $\varepsilon_{1} M_{1}^{2}=\mu^{2} / \lambda_{1} \sim$ $\left(10^{2} \mathrm{GeV}\right)^{2}$.

$V_{2}(\Phi, h, \varphi)$ part causes the chiral symmetry breaking, e.g., $\mathrm{SU}(2)_{\mathrm{L}} \times \mathrm{SU}(2)_{\mathrm{R}} \rightarrow \mathrm{SU}(2)_{\mathrm{V}}$. Using the $2 \times 2$ matrix scalar field $\varphi=\sigma+i \tau \cdot \pi$ (chiral sigma-model field), we may similarly write the potential

$$
V_{2}(\Phi, h, \varphi)=\frac{1}{4} \lambda_{2}\left(\operatorname{tr}\left(\varphi^{\dagger} \varphi\right)-\varepsilon_{2} \Phi_{1}^{2}\right)^{2}+V_{\text {break }}(\Phi, h, \varphi)
$$


with another small parameter $\varepsilon_{2} \simeq 10^{-34}$. The first term reproduces the linear $\sigma$-model potential invariant under the chiral $\mathrm{SU}(2)_{\mathrm{L}} \times \mathrm{SU}(2)_{\mathrm{R}}$ transformation $\varphi \rightarrow g_{\mathrm{L}} \varphi g_{\mathrm{R}}$ when $\varepsilon_{2} \Phi_{1}^{2}$ is replaced by the VEV $\varepsilon_{2} M_{1}^{2}=m^{2} / \lambda_{2}$. The last term $V_{\text {break }}$ stands for the chiral symmetry breaking term which is caused by the explicit quark mass terms appearing as the result of tiny Yukawa couplings of $u, d$ quarks, $y_{u}, y_{d}$, to the Higgs doublet $h$; e.g.,

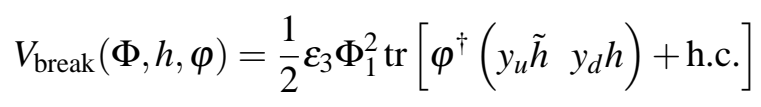

with $\varepsilon_{3} \sim 4 \pi \varepsilon_{2}$ and $\tilde{h} \equiv i \sigma_{2} h^{*}$.

\section{2 quantum mechanically}

As soon as we come to quantum field theory, we are confronted with the SI anomaly:

$$
\text { Scale invariance suffers from an Anomaly. }
$$

Usual wisdom tells us so. Owing to the UV divergence in quantum field theory, it is necessary to introduce a (dimensionful) renormalization point $\mu$, which necessarily break the classical SI. If we also take the renormalization point $\mu$ into account, the dimension counting identity comes to read

$$
\left(\mu \frac{\partial}{\partial \mu}+\sum_{i} \phi_{i} \frac{\partial}{\partial \phi_{i}}\right) V(\phi)=4 V(\phi) .
$$

The anomaly $\mu(\partial / \partial \mu) V$ term may be eliminated by using renormalization group equation (RGE):

$$
\left(\mu \frac{\partial}{\partial \mu}+\sum_{a} \beta_{a}(\lambda) \frac{\partial}{\partial \lambda_{a}}+\sum_{i} \gamma_{i}(\lambda) \phi_{i} \frac{\partial}{\partial \phi_{i}}\right) V(\phi)=0
$$

Then, we obtain

$$
\left(\sum_{i}\left(1-\gamma_{i}(\lambda)\right) \phi_{i} \frac{\partial}{\partial \phi_{i}}-\sum_{a} \beta_{a}(\lambda) \frac{\partial}{\partial \lambda_{a}}\right) V(\phi)=4 V(\phi),
$$

which replaces the above naive dimension counting equation $\sum_{i} \phi_{i}\left(\partial / \partial \phi_{i}\right) V(\phi)=4 V(\phi)$. With either Eq. (3.6) or Eq. (3.7), we cannot conclude the vanishing potential value $V\left(\phi^{0}\right)=0$ at the stationary point $\phi^{0}$. Eq. 3.7) shows that the anomalous dimension $\gamma_{i}(\lambda)$ is not the problem, but $\beta_{a}(\lambda)$ terms may be problematic.

Still, if we assume the existence of Infrared Fixed Points $\beta_{a}\left(\lambda_{\mathrm{IR}}\right)=0$ and that the theory on top of that point $\lambda_{\mathrm{IR}}$ is well-defined, then, I can prove that the potential value $V\left(\phi^{0}\right)$ at the stationary point $\phi=\phi^{0}$ is zero at any finite $\mu$ (not necessarily in the IR limit $\mu \rightarrow 0$ ). That is, the vanishing property of the stationary potential value $V(\phi)$ is not injured by the scale-invariance anomaly[14].

Even then, however, we will meet the same difficulty - flat direction problem - as that we will encounter also in the next approach which we discuss from now on. So we do not discuss this approach assuming the IR fixed point anymore here. 


\section{3 quantum scale invariant renormalization}

Shaposhnikov and Zenhausern [2] proposed a new approach to this anomaly obstacle for the scale invariance scenario. Their proposal is based on a very simple observation that SI can be maintained even in quantum field theory if we have a dilaton field $\Phi$ in the system. Generally, if a regularization method exists which keeps a symmetry, then it implies the absence of anomaly for the symmetry. In this case of SI, the extension to $n$-dimension is shown possible keeping SI if a dilaton field $\Phi$ is used, as we explain shortly.

This way of quantum SI renormalization is, however, not new, but actually has long been known since the original proposal by Englert, Truffin and Gastmans[1]. It was also used in the prior scale invariant approaches to the $\mathrm{CC}$ problem [3] 4 .

Recall the way how the scalar quartic coupling $\lambda$ and Yukawa coupling $y$ are kept dimensionless in $n$ dimension in the usual dimensional regularization. It is realized by introducing renormalization scale $\mu$ as follows:

\section{Usual dimensional regularization}

$$
\begin{array}{llll}
\lambda\left(h^{\dagger}(x) h(x)\right)^{2} & \rightarrow & \lambda \mu^{4-n}\left(h^{\dagger}(x) h(x)\right)^{2} & {[h]=\frac{n-2}{2}} \\
y \bar{\psi}(x) \psi(x) h(x) & \rightarrow & y \mu^{\frac{4-n}{2}} \bar{\psi}(x) \psi(x) h(x) & {[\psi]=\frac{n-1}{2}}
\end{array}
$$

To avoid the introduction of explicit dimensionful parameter $\mu$ violating the SI, we can replace $\mu$ by a power of the dynamical dilaton field $\Phi(x), \Phi^{\frac{2}{n-2}}(x)$, of dimension 1 as

$$
\begin{aligned}
& \text { SI prescription } \\
& \lambda\left(h^{\dagger}(x) h(x)\right)^{2} \rightarrow \lambda\left[\Phi(x)^{\frac{2}{n-2}}\right]^{4-n}\left(h^{\dagger}(x) h(x)\right)^{2} \\
& y \bar{\psi}(x) \psi(x) h(x) \rightarrow y\left[\Phi(x)^{\frac{2}{n-2}}\right]^{\frac{4-n}{2}} \bar{\psi}(x) \psi(x) h(x) .
\end{aligned}
$$

Since no dimensionful parameter is introduced, this prescription really keeps SI in any dimension $n$. But the price we have to pay is the non-renormalizable interaction terms; that is, on the vacuum in which the dilaton field develops the $\operatorname{VEV}\langle\Phi(x)\rangle=M$, the introduced fractional power of $\Phi(x)$ yields non-polynomial "evanescent" interactions $\propto 2 \varepsilon=4-n$ :

$$
\Phi(x)=M+\phi(x) \rightarrow[\Phi(x)]^{\frac{4-n}{n-2}}=M^{\frac{\varepsilon}{1-\varepsilon}}\left(1+\frac{\varepsilon}{1-\varepsilon} \frac{\phi(x)}{M}++\frac{1}{2} \frac{\varepsilon(2 \varepsilon-1)}{(1-\varepsilon)^{2}} \frac{\phi(x)^{2}}{M^{2}}+\cdots\right) .
$$

This prescription gives quantum scale invariant theory, which might realize the vanishing CC. So let us examine this theory in more detail.

\section{Quantum Scale-Invariant Renormalization: 2-scalar model}

Explicit calculations were performed by Ghilencea and his collaborators [15, 16, 17 in a simple 2-scalar model; following Ref. [15], we henceforth use notations $\phi(x)$ and $\sigma(x)$ to denote our Higgs field $h(x)$ and dilaton field $\Phi(x)(h \rightarrow \phi, \Phi \rightarrow \sigma)$. Then the Lagrangian reads

$$
\mathscr{L}=\frac{1}{2} \partial_{\mu} \phi \cdot \partial^{\mu} \phi+\frac{1}{2} \partial_{\mu} \sigma \cdot \partial^{\mu} \sigma-V(\phi, \sigma)
$$


with scale-invariant potential in $n$ dimension:

$$
V(\phi, \sigma)=\mu(\sigma)^{4-n}\left(\frac{\lambda_{\phi}}{4} \phi^{4}-\frac{\lambda_{m}}{2} \phi^{2} \sigma^{2}+\frac{\lambda_{\sigma}}{4} \sigma^{4}\right)
$$

with

$$
\mu(\sigma)=z \sigma^{\frac{2}{n-2}} .
$$

Here $z$ is a renormalization point parameter introduced by Tamarit [18 to discuss renormalization group equation (RGE) in this quantum SI theory, but we can take $z=1$ if we do not care about RGE. At tree level, $\lambda_{m}^{2}=\lambda_{\phi} \lambda_{\sigma}$ is assumed so that the potential becomes a complete square form:

$$
\begin{gathered}
V(\phi, \sigma)=\mu(\sigma)^{4-n} \frac{\lambda_{\phi}}{4}\left(\phi^{2}-\varepsilon \sigma^{2}\right)^{2}, \\
\text { with } \quad \lambda_{m}=\varepsilon \lambda_{\phi}, \quad \lambda_{\sigma}=\varepsilon^{2} \lambda_{\phi} .
\end{gathered}
$$

Note: If the dilaton $\sigma$ and the Higgs $\phi$ are supposed to get the VEVs of order of the Planck scale mass $M \sim 10^{18} \mathrm{GeVand}$ the electroweak mass $\mu \sim 10^{2} \mathrm{GeV}$, respectively, then the parameter $\varepsilon=\langle\phi\rangle^{2} /\langle\sigma\rangle^{2}$ is very tiny $\sim 10^{-32}$. We know that Higgs quartic coupling $\lambda=2 \lambda_{\phi} \sim 1 / 4$ so that $\lambda_{m}$ and $\lambda_{\sigma}$ are very tiny of $O(\varepsilon)$ and $O\left(\varepsilon^{2}\right)$, respectively.

Ghilencea has shown the following for this quantum scale invariant theory:

1. Non-renormalizability: higher and higher order non-polynomial interaction terms of the form

$$
\frac{\phi^{4+2 p}}{\sigma^{2 p}} \quad(p=1,2,3, \cdots)
$$

are induced by the evanescent interactions at higher loop level (up to $p \leq \ell$ at $\ell$ loop level), and they must also be included as counterterms. These terms, however, can be neglected in the low-energy region below Planck scale $E\langle\langle\sigma\rangle \sim M$. So the usual renormalizable theory is an effective low energy theory valid below Planck energy, irrespectively of whether the gravity is quantized or not.

2. Mass hierarchy is stable: If we put

$$
\lambda_{\phi}=\bar{\lambda}_{\phi}, \quad \lambda_{m}=\varepsilon \bar{\lambda}_{m}, \quad \lambda_{\sigma}=\varepsilon^{2} \bar{\lambda}_{\sigma}
$$

with $O(1)$ coupling constants $\bar{\lambda}_{i}(i=\phi, m, \sigma)$ and very tiny $\varepsilon=10^{-32}$, then, $\bar{\lambda}_{i}$ 's remain of $O(1)$ stably against radiative corrections. This is essentially because $\sigma^{2} \phi^{2}$ term comes only through the $\lambda_{m} \phi^{2} \sigma^{2}$ interaction.

Explicit form of the one-loop potential at $n=4$ is actually given in the Scale Invariant form:

$$
\begin{gathered}
V(\phi, \sigma)=\frac{\lambda_{\phi}}{4} \phi^{4}-\frac{\lambda_{m}}{2} \phi^{2} \sigma^{2}+\frac{\lambda_{\sigma}}{4} \sigma^{4} \\
+\frac{\hbar}{64 \pi^{2}}\left\{M_{1}^{4}\left(\ln \frac{M_{1}^{2}}{z^{2} \sigma^{2}}-\frac{3}{2}\right)+M_{2}^{4}\left(\ln \frac{M_{2}^{2}}{z^{2} \sigma^{2}}-\frac{3}{2}\right)+\Delta V\right\}, \\
\Delta V=-\lambda_{\phi} \lambda_{m} \frac{\phi^{6}}{\sigma^{2}}+\left(16 \lambda_{\phi} \lambda_{m}-6 \lambda_{m}^{2}+3 \lambda_{\phi} \lambda_{\sigma}\right) \phi^{4} \\
+\left(-16 \lambda_{m}+25 \lambda_{\sigma}\right) \lambda_{m} \phi^{2} \sigma^{2}-21 \lambda_{\sigma}^{2} \sigma^{4}
\end{gathered}
$$


where $M_{i}^{2}(i=1,2)$ are two mass-square eigenvalues for two scalar fields around the VEVs $\phi$ and $\sigma$, so $M_{i}^{2} / \sigma^{2}$ are dimensionless functions of dimensionless variable $\phi^{2} / \sigma^{2}$. The $\Delta V$ potential is the finite part which comes from the $O(\varepsilon)$ evanescent interaction terms multiplied by the one-loop divergence $1 / \varepsilon$.

However, there is a problem to which Ghilencea has not mentioned:

3. Vanishing CC again requires fine tuning! owing to quantum corrections.

This is the most important point in this paper, so let us now explain it in detail. Since $V(\phi, \sigma)$ is a dimension-4 function in $\sigma$ and $\phi$, it takes the form

$$
V(\phi, \sigma)=\sigma^{4} W(x) \quad \text { with } \quad x \equiv \phi^{2} / \sigma^{2} .
$$

Since the stationarity conditions

$$
\left\{\begin{array}{l}
\phi \frac{\partial}{\partial \phi} V=\quad \sigma^{4} W^{\prime}(x) \cdot 2 x=0 \\
\sigma \frac{\partial}{\partial \sigma} V=\sigma^{4}\left(4 W(x)+W^{\prime}(x) \cdot(-2 x)\right)=0
\end{array}\right.
$$

requires both

$$
W^{\prime}(x)=0 \quad \text { and } \quad W(x)=0 \quad \text { are satisfied, unless } \sigma=\phi=0 .
$$

Let us examine these conditions with the above 1-loop potential [4.7):

$$
\begin{aligned}
W(x)= & \frac{\lambda_{\phi}}{4} x^{2}-\frac{\lambda_{m}}{2} x+\frac{\lambda_{\sigma}}{4} \\
+ & \frac{\hbar}{64 \pi^{2}}\left\{\frac{M_{1}^{4}}{\sigma^{4}}\left(\ln \frac{M_{1}^{2}}{z^{2} \sigma^{2}}-\frac{3}{2}\right)+\frac{M_{2}^{4}}{\sigma^{4}}\left(\ln \frac{M_{2}^{2}}{z^{2} \sigma^{2}}-\frac{3}{2}\right)\right. \\
& \left.\quad-\lambda_{\phi} \lambda_{m} x^{3}+\left(16 \lambda_{\phi} \lambda_{m}-6 \lambda_{m}^{2}+3 \lambda_{\phi} \lambda_{\sigma}\right) x^{2}+\left(-16 \lambda_{m}+25 \lambda_{\sigma}\right) \lambda_{m} x-21 \lambda_{\sigma}^{2}\right\} .
\end{aligned}
$$

First consider these conditions at tree level; the stationary point $x=x_{0}$ should satisfy

$$
\left\{\begin{aligned}
W^{\prime}\left(x_{0}\right)=\frac{\lambda_{\phi}}{2} x_{0}-\frac{\lambda_{m}}{2}=0 & \rightarrow x_{0}=\frac{\lambda_{m}}{\lambda_{\phi}}, \\
W\left(x_{0}\right)=\frac{\lambda_{\phi}}{4} x_{0}^{2}-\frac{\lambda_{m}}{2} x_{0}+\frac{\lambda_{\sigma}}{4}=0 & \rightarrow \lambda_{\sigma}=\frac{\lambda_{m}^{2}}{\lambda_{\phi}} .
\end{aligned}\right.
$$

Note here that the stationary point $x_{0}=\frac{\langle\phi\rangle^{2}}{\langle\sigma\rangle^{2}}$ is already determined by the first condition $W^{\prime}\left(x_{0}\right)=0$ alone, while the second one $W(x)=0$ imposes a constraint on the coupling constants $\lambda_{i}$ 's. This constraint $\lambda_{\sigma} \lambda_{\phi}=\lambda_{m}^{2}$ at this stage is the condition we have initially imposed on the tree potential in Eq. (4.4).

At one-loop level, next, the stationary point may be shifted and the coupling constants may be adjusted:

$$
x=x_{0}+\hbar x_{1}, \quad \lambda_{i} \Rightarrow \lambda_{i}+\hbar \delta \lambda_{i}(i=\phi, m, \sigma) .
$$


The first condition $W^{\prime}(x)=0$ requires, for $O(\hbar)$ parts,

$$
\begin{aligned}
\left.W^{\prime}(x)\right|_{O(\hbar)}= & \frac{\lambda_{\phi}}{2} x_{1}+\frac{\delta \lambda_{\phi}}{2} x_{0}+\frac{\delta \lambda_{m}}{2} \\
& +\frac{1}{64 \pi^{2}}\left[4 \lambda_{\phi} \lambda_{m}\left(3+2 x_{0}-x_{0}^{2}\right)\left(\ln \frac{2 \lambda_{m}\left(1+x_{0}\right)}{z^{2}}-1\right)+16 \lambda_{m}^{2}\left(1+x_{0}\right)\right] .
\end{aligned}
$$

This determines, as at tree level, the VEV's shift at 1-loop level $\hbar x_{1}$. We may or may not adjust the coupling constants at this stage. But important is the point that this condition (4.14) is consistent with the VEV (mass) hierarchy; that is, no fine tuning of the coupling constants is necessary to maintain the tiny ratio of field VEVs $x=\langle\phi\rangle^{2} /\langle\sigma\rangle^{2}$ at tree level, $x_{0}=\lambda_{m} / \lambda_{\phi}=\varepsilon \sim 10^{-32}$. Indeed, every term in Eq. (4.14) is $O(\varepsilon)$ simply by keeping the order of magnitude of the coupling constants as in Eq. (4.6); namely, the barred coupling constants are all kept of order $1, \bar{\lambda}_{i} \sim \delta \bar{\lambda}_{i} \sim O(1)$, and then the VEV ratio $x_{0}+\hbar x_{1}$ consistently remains of $O(\varepsilon)$ :

$$
\lambda_{m}, \delta \lambda_{m} \sim O(\varepsilon), \lambda_{\phi}, \delta \lambda_{\phi} \sim O(1) \rightarrow x_{0}, x_{1} \sim O(\varepsilon) \rightarrow x=\frac{\langle\phi\rangle^{2}}{\langle\sigma\rangle^{2}}=x_{0}+\hbar x_{1} \sim O(\varepsilon) .
$$

Next is the second condition:

$$
\left.W(x)\right|_{O(\hbar)}=\frac{\delta \lambda_{\phi}}{4} x_{0}^{2}+\frac{\delta \lambda_{m}}{2} x_{0}+\frac{\delta \lambda_{\sigma}}{4}+\frac{1}{64 \pi^{2}}\left[4 \lambda_{m}^{2}\left(1+x_{0}\right)^{2}\left(\ln \frac{2 \lambda_{m}\left(1+x_{0}\right)}{z^{2}}-\frac{3}{2}\right)\right] .
$$

Again, this gives a constraint on the coupling constants. Here again all the terms are consistently of $O\left(\varepsilon^{2}\right)$, so that $W(x)=0$ can be realized up to $o\left(\varepsilon^{2}\right)$ by $O(1)$ tuning of the barred coupling constants $\bar{\lambda}_{\phi}, \bar{\lambda}_{m}, \bar{\lambda}_{\sigma}$. However, although $W(x)$ at the stationary point can be made vanish very precisely in the order as tiny as $\varepsilon^{2} \sim 10^{-64}$, the Vacuum Energy $V=\sigma^{4} W(x)$ itself vanishes only in the sense of $O\left(\varepsilon^{2}\right) \times \sigma^{4}=O\left((100 \mathrm{GeV})^{4}\right)$. This is because the Planck energy is so huge; $\langle\sigma\rangle^{4}=$ $\left(10^{18} \mathrm{GeV}\right)^{4}=10^{64} \times(100 \mathrm{GeV})^{4}$. If we require the vanishingness up to the order of presently observed vacuum energy $\Lambda_{0} \sim(1 \mathrm{meV})^{4} \sim 10^{-56} \times(100 \mathrm{GeV})^{4}$, then, we have still to tune the barred coupling constants $\bar{\lambda}_{\phi}, \bar{\lambda}_{m}, \bar{\lambda}_{\sigma}$ in 56 digits! We still need superfine tuning even in quantum scale-invariant theory. This is nothing but reappearance of the original CC problem! We have to conclude:

$$
\text { Quantum SI is not enough to solve the CC problem. }
$$

Shaposhnikov and Zenhausern[2] noted that there are degrees of freedom of coupling constants to realize the condition $W(x)=0$, but they did not recognize that it requires the superfine tuning of the coupling constants which is essentially the same problem as the CC problem we originally wanted to solve.

Note also, however, that this is in fact the problem beyond the perturbation theory. We are discussing the vacuum energy in much finer precision than the perturbation (loop) expansion parameter $\hbar / 16 \pi^{2} \sim 1 / 158$.

\section{What happens?}

If the theory is quantum scale-invariant, then we have the dimension counting equation

$$
\sum_{i} \phi_{i} \frac{\partial}{\partial \phi_{i}} V(\phi)=4 V(\phi)
$$


which implies $V\left(\phi_{i}^{0}\right)=0$ at any stationary point $\phi_{i}^{0}$, and any point in that direction, $\rho \phi_{i}^{0}$ with $\forall \rho \in \mathbf{R}$ also realizes the vanishing energy $V\left(\rho \phi_{i}^{0}\right)=\rho^{4} V\left(\phi_{i}^{0}\right)=0$ (flat direction). Conversely speaking, therefore, if $V(\phi) \neq 0$ at $\exists \phi$, then the potential is not stationary at that $\phi$.

In the above: $V(\phi, \sigma)=\sigma^{4} W(x)$ was flat in the direction $\phi^{2} / \sigma^{2}=x_{0}$ at tree level, $W\left(x_{0}\right)=0$, but, at one-loop, the potential did not exactly satisfy $W\left(x_{0}+\hbar x_{1}\right)=0$ at the 'stationary point' realizing $W^{\prime}\left(x_{0}+\hbar x_{1}\right)=0$ exactly, unless the coupling constants were superfine-tuned. The value $W\left(x_{0}+\hbar x_{1}\right)$ is just as tiny as $\varepsilon^{2} \sim 10^{-64}$ but not exactly zero.

This means from the above Eq. (4.10) that the point $x_{0}+\hbar x_{1}$ realizes the stationarity with respect to $\phi$ but not necessarily to $\sigma$. " $W\left(x_{0}+\hbar x_{1}\right) \neq$ exactly 0 " means that the potential has a tiny gradient $\sigma(\partial / \partial \sigma) V=4 \sigma^{4} W(x)=\sigma^{4} O\left(\varepsilon^{2}\right) \neq 0$ in the $\sigma$-direction (or, more precisely, $\phi^{2} / \sigma^{2}=$ $x_{0}+\hbar x_{1}$ direction) and that the potential is actually stationary only at the origin $\sigma=0$ ! That is,

$$
\text { The flat direction is lifted by the radiative correction. }
$$

Quantum scale invariance alone does not protect the flat direction, automatically. Artificial superfine tuning of the coupling constants was required to keep the flat direction.

Tomboulis [4] however proposed an interesting mechanism with which the condition $W(x)=0$ may automatically be satisfied without any fine tuning of the coupling constants. Let us explain his arguments. Recall that the stationarity $\frac{\delta V}{\delta \phi}=0$ and $\frac{\delta V}{\delta \sigma}=0$ required, for $V=\sigma^{4} W$, respectively,

$$
\begin{aligned}
\text { (1) }\left.W^{\prime}(x ; \lambda)\right|_{x=x_{0}(\lambda)}=0 \quad & \quad \text { (2) } \quad W\left(x_{0}(\lambda) ; \lambda\right)=0 \\
\text { determines the VEV ratio } x=\frac{\phi^{2}}{\sigma^{2}}=x_{0}(\lambda) & \text { demands super fine tuning of } \lambda \text { 's. }
\end{aligned}
$$

Tomboulis introduced a renormalization point $\mu$ in addition to the dilaton field $\sigma$ and consider the running of coupling constant: ${ }^{1}$

$$
\bar{\lambda}(z), \quad z \equiv \frac{\mu}{\sigma}: \quad \text { renormalization point parameter . }
$$

Then, he claims that, the second condition in Eq. 5.3, now reading

$$
W\left(x_{0}(\bar{\lambda}(z)) ; \bar{\lambda}(z)\right)=0,
$$

is simply an equation determining the renormalization point $z_{0}=\mu_{0} / \sigma_{0}$ and so is automatically satisfied without any fine tuning.

This interesting idea, however, does not work unfortunately, since

$$
\frac{d}{d z} W\left(x_{0}(\bar{\lambda}(z)) ; \bar{\lambda}(z)\right)=0 .
$$

Changing the renormalization point $z=\mu / \sigma$ cannot change the value of $W\left(x_{0}(\bar{\lambda}(z)) ; \bar{\lambda}(z)\right)$ since it is a physical quantity independent of the choice of renormalization point.

\footnotetext{
${ }^{1}$ The coupling constants run even in quantum scale invariant theory despite the fact that the usual beta functions $\beta_{a}(\lambda)$ represent the anomaly for the scale invariance. Tomboulis as well as Shaposhnikov-Zenhausern did know this fact, but it was fully clarified by Tamarit 18 and properly used by Ghilencea[15. Here we use the letter $z$ following Ghilencea 15] to denote the renormalization point parameter $\xi$ of Tamarit's [18.
} 
We thus have no mechanism which can preserve the flat direction against quantum radiative corrections. We still need another symmetry to realize the flat direction. Supersymmetry (SUSY) would be an immediate candidate for it. But it will also introduce another problem how to break it spontaneously. Depending on the way of breaking, the superfine tuning problem may reappear.

\section{Discussions: hierarchy problem}

Although the quantum scale invariance is not yet sufficient for solving the CC problem, it should be emphasized that it already almost solved the hierarchy problem.

There are two aspects of the hierarchy problem:

1. Origin: to explain the origin why the hierarchy exist.

2. Stability: to explain its stability against radiative corrections, once it exists anyway.

\section{1 stability against radiative corrections}

As for the stability against the radiative correction, it is guaranteed, for instance, by SUSY as a well-known example. If the system has scale invariance, there exist only the logarithmic divergences but no quadratic divergences, so that the stability is automatic in the SM, as was emphasized by Bardeen[19] in 1980's. In the present two scalar model, however, if the $\phi^{2} \sigma^{2}$ term is radiatively induced with $O(1)$ coefficient, the (mass) hierarchy is immediately broken since $\langle\sigma\rangle=M$ is of Planck or GUT energy scale. We have observed based on Ghilencea's explicit computation that the coupling constant $\lambda_{m}$ of the $\phi^{2} \sigma^{2}$ term remains of order $O(\varepsilon)$ at one-loop. The general reason for it is that the coupling between $\phi^{2}$ and $\sigma^{2}$ totally disappears in the 2-scalar model if $\lambda_{m}=\varepsilon \bar{\lambda}_{m}$ vanishes at tree level, so it must be proportional to $\varepsilon$ at any loop level[2].

If the gravity interaction is taken into account, we need additional reasoning, since $\phi^{2}$ and $\sigma^{2}$ can couple through gravity without factor $\lambda_{m}$. The point is that $\sigma^{2}$ can couple to the gravity loop directly via $\sqrt{-g} \sigma^{2} R$ whereas $\phi^{2}$ can couple to it only through the kinetic term $\sqrt{-g} g^{\mu v} \partial_{\mu} \phi \partial_{\nu} \phi$ unless we use the $O(\varepsilon) \lambda_{m}$ interaction $\sqrt{-g} \lambda_{m} \phi^{2} \sigma^{2}$. If we use the kinetic term interaction vertex in which $\phi$ is accompanied by a derivative, the gravity loop graph will induce the term of the form $\sqrt{-g} \sigma^{2} \partial_{\mu} \phi \partial^{\mu} \phi$. It is of dimension 6 and should be divided by a mass dimension-2 quantity $M^{2}$. But we have no such a dimensionful parameter in this quantum scale invariant theory and only field that can appear in the denominator is the dilaton field $\sigma^{2}$, so it eventually gives just the Higgs kinetic term.

Another worry is whether the gravity loop might induce the $\sigma^{4}$ term with $O(1)$ or even $O(\varepsilon)$ or not. The worry is only the gravity loops and only the term $\sqrt{-g} \sigma^{2} R$ is relevant. Then $\sigma^{2}$ plays the role of an overall factor in front of the action just like inverse of the Planck constant, $1 / \hbar$. So the $L$-loop gravity graphs can produce only the term proportional to $\left(\sigma^{2}\right)^{1-L}$, and hence no positive power of $\sigma^{2}$ terms are induced by gravity loops.

\section{2 origin of the hierarchy}

In the above wishful scenario in Section 3.1, we have "realized" large gauge hierarchies simply by assuming tiny parameters $\varepsilon_{1} \simeq 10^{-28}$ and $\varepsilon_{2} \simeq 10^{-34}$ :

$$
V_{1}=\frac{1}{2} \lambda_{2}\left(h^{\dagger} h-\varepsilon_{1} \Phi_{1}^{2}\right)^{2} \quad \text { and } \quad V_{2} \supset \frac{1}{4} \lambda_{2}\left(\operatorname{tr}\left(\varphi^{\dagger} \varphi\right)-\varepsilon_{2} \Phi_{1}^{2}\right)^{2} .
$$


However, the chiral symmetry breaking scale $\varepsilon_{2}\left\langle\Phi_{1}\right\rangle$, for instance, can usually be explained by the running coupling as follows; if GUT is assumed, the SU(3) gauge coupling $\alpha_{3}=g_{3}^{2} / 4 \pi$ at scale $\sqrt{\varepsilon_{0}} M \equiv M_{1}$ evolves as $\alpha_{3}(\mu)$ a la RGE as the scale $\mu$ changes, and reaches to the $O(1)$ critical coupling $\alpha_{3}^{\mathrm{cr}} \simeq 1$ at scale $\mu \simeq \Lambda_{\mathrm{QCD}}$ to break the chiral symmetry, so that $\sqrt{\varepsilon_{2}} M_{1} \simeq \Lambda_{\mathrm{QCD}}$. Thus the relation between the GUT scale $M_{1}$ and QCD scale $\Lambda_{\mathrm{QCD}}$ is fixed by the running gauge coupling constant $\alpha_{3}\left(M_{1}\right)$ at scale $M_{1}$ as

$$
\begin{aligned}
\mu \frac{d}{d \mu} \alpha_{3}(\mu)=2 b_{3} \alpha_{3}^{2}(\mu) & \rightarrow \frac{1}{\alpha_{3}(\mu)}=\frac{1}{\alpha_{3}\left(M_{1}\right)}-b_{3} \ln \frac{\mu^{2}}{M_{1}^{2}} \\
& \rightarrow \frac{1}{\alpha_{3}^{\mathrm{cr}}}=\frac{1}{\alpha_{3}\left(M_{1}\right)}-b_{3} \ln \frac{\Lambda_{\mathrm{QCD}}^{2}}{M_{1}^{2}}
\end{aligned}
$$

where $\alpha_{3}^{\text {cr }}=O(1)$ quantity like $\pi / 3$, so explains the huge hierarchy:

$$
\varepsilon_{2}=\frac{\Lambda_{\mathrm{QCD}}^{2}}{M_{1}^{2}}=\exp \frac{1}{b_{3}}\left(\frac{1}{\alpha_{3}\left(M_{1}\right)}-\frac{1}{\alpha_{3}^{\mathrm{cr}}}\right) .
$$

This is the usual explanation.

In quantum SI theory, $\alpha_{3}\left(M_{1}\right)$ here, probably, should be replaced by $M_{1}$-independent initial gauge coupling $\alpha_{3}^{\text {init }}$, while the initial scale $M_{1}^{2}$ should be replaced by the dilaton field VEV $\langle\sigma\rangle^{2}$. Then

$$
\frac{1}{\alpha_{3}^{\mathrm{cr}}}-\frac{1}{\alpha_{3}^{\text {init }}}=-b_{3} \ln \frac{\Lambda_{\mathrm{QCD}}^{2}}{\langle\sigma\rangle^{2}}
$$

so that the QCD scale $\Lambda_{\mathrm{QCD}}$ is always scaled with the dilaton $\operatorname{VEV}\langle\sigma\rangle$.

This hierarchy should show up in the effective potential. Since $\Lambda_{\mathrm{QCD}}^{2}$ here should stand for the $\operatorname{VEV} \varphi^{\dagger} \varphi$ of the chiral sigma model scalar field $\varphi$, we suspect that we should be able to derive an effective potential of quasi Coleman-Weinberg type like

$$
V(\sigma, \varphi)=\frac{\left(\varphi^{\dagger} \varphi\right)^{2}}{64 \pi^{2}}\left(-b_{3} \ln \frac{\varphi^{\dagger} \varphi}{\sigma^{2}}+\frac{1}{\alpha_{3}^{\text {init }}}-\frac{1}{\alpha_{3}^{\text {cr }}}\right)^{2} .
$$

Note that this form of SI potential is devised such that it has a non-vanishing field stationary point at $\langle\varphi\rangle^{\dagger}\langle\varphi\rangle /\langle\sigma\rangle^{2}=x_{0}$ satisfying $b_{3} \ln x_{0}=\left(\alpha_{3}^{\text {init }}\right)^{-1}-\left(\alpha_{3}^{\text {cr }}\right)^{-1}$.

\section{Conclusion}

I have shown in this talk that the scale invariance gives a natural mechanism for guaranteeing the vanishing vacuum energy at each step of hierarchical successive spontaneous symmetry breakings, at least in the classical field theory. I also explained the Englert-Truffin-Gastmans prescription which, I called quantum scale-invariant renormalization, preserves the scale-invariance also in quantum field theory. Even with such a prescription, however, the radiative corrections lift the flat directions of the potential and leave only the origin $\phi_{i}=0$ in field space as the stationary point, unless the superfine tuning of the coupling constants is made. Therefore, the scale-invariance alone is not sufficient for realizing the vanishing vacuum energy. 
I believe that the scale invariance is the right direction for solving the $\mathrm{CC}$ problem, but something is still missing. We need yet another symmetry or a mechanism to realize

$$
\begin{aligned}
\text { Spontaneous SI breaking } & =\text { Non-vanishing field VEV } \\
& =\exists \text { flat direction of } V(\phi) .
\end{aligned}
$$

\section{Acknowledgments}

I would like to thank Jisuke Kubo, Ichiro Oda and Dumitru Ghilencea for valuable discussions on scale invariant theories. I also owe to Makoto Kobayashi, Naoshi Sugiyama and Misao Sasaki for discussions on thermal history of the universe in the early stage of this work. I am also grateful to Toshihide Maskawa, Nobuyoshi Ohta, Ikuo Sogami, Shotaro Shiba, Naoki Yamatsu, Masato Yamanaka and other colleagues at Maskawa Institute, Kyoto Sangyo University for critical discussions and encouragement. This work is supported in part by Japan Society for the Promotion of Science (JSPS) Grant-in-Aid for Scientific Research (C) Grant Number JP18K03659.

\section{References}

[1] F. Englert, C. Truffin and R. Gastmans, Nucl. Phys. B 117 (1976) 407.

[2] M. Shaposhnikov and D. Zenhausern, Phys. Lett. B 671 (2009) 162.

[3] I. Antoniadis and N. C. Tsamis, Phys. Lett. 144B (1984) 55.

[4] E. T. Tomboulis, Nucl. Phys. B 329 (1990) 410.

[5] C. Wetterich, Nucl. Phys. B 302 (1988) 668.

[6] M. Shaposhnikov and D. Zenhausern, Phys. Lett. B 671 (2009) 187.

[7] E. Rabinovici, B. Saering and W. A. Bardeen, Phys. Rev. D 36 (1987) 562.

[8] K. A. Meissner and H. Nicolai, Phys. Lett. B 648 (2007) 312; Phys. Lett. B 660 (2008) 260.

[9] P. G. Ferreira, C. T. Hill and G. G. Ross, Phys. Lett. B 763 (2016) 174; Phys. Rev. D 95 (2017) no.4, 043507; Phys. Rev. D 98 (2018) no.11, 116012.

[10] I. Oda, Adv. Stud. Theor. Phys. 13 (2019) 195

[11] N. C. Tsamis and R. P. Woodard, Annals Phys. 168 (1986) 457.

[12] D. M. Ghilencea, JHEP 1903 (2019) 049

[13] I. Oda, Adv. Stud. Theor. Phys. 14 (2020) no.1-4, 9

[14] T. Kugo, "Cosmological Constant Problem and Scale Invariance", Talk given in the 23rd International Summer Institute on Phenomenology of Elementary Particle Physics and Cosmology (SI2017), August 25 - 31, 2017, Fuji-Yoshida, Japan [Slide at SI2017 Website: http://muse.sc.niigata-u.ac.jp/ si17/]

[15] D.M. Ghilencea, Phys.Rev. D93(2016)105006.

[16] Ghilencea, Lalak and Olszewski, Eur.Phys.J. C(2016)76:656.

[17] Ghilencea, Phys.Rev. D97(2018)075015.

[18] C. Tamarit, JHEP 12(2013)098.

[19] W. A. Bardeen, "On naturalness in the standard model," FERMILAB-CONF-95-391-T. 